\title{
Automatisierte Kalibrierung eines Gonioreflektometers zum Messen der BRDF
}

\author{
Andreas W. Winkler, Bernhard G. Zagar \\ Johannes Kepler Universität Linz \\ Institut für Elektrische Messtechnik \\ Altenbergerstraße 69 \\ 4040 Linz, Österreich
}

\section{Zusammenfassung}

Ein Gonioreflektometer ist ein Gerät zum Messen der Bidirektionalen Reflektanzverteilungsfunktion (BRDF) und besteht im Allgemeinen aus einer Lichtquelle, einem lichtempfindlichen Detektor und einer Aufnahme für die Probe. Für ein solches, im Labor aufgebautes Gerät werden zwei Kalibrierverfahren vorgestellt: Eines zur Bestimmung der intrinsischen Parameter der als Detektor verwendeten Kamera, der Konstruktionsparameter des als Probenaufnahme verwendeten sphärischen Gelenks und der relativen Lage und Orientierung zwischen Kamera und Gelenk und ein zweites Verfahren zur Bestimmung der Position der Lichtquelle. Beide Verfahren verwenden ein eigenes Kalibriertarget, bieten eine hohe Genauigkeit und können jeweils vollständig automatisiert arbeiten.

Keywords: Bidirektionale Reflektanzverteilungsfunktion, Gonioreflektometer, Kalibrierung, Kameramodell, Denavit-Hartenberg Notation

\section{Einleitung}

Die Bidirektionale Reflektanzverteilungsfunktion (BRDF) [1]

$$
f\left(\varphi_{\mathrm{i}}, \theta_{\mathrm{i}} ; \varphi_{\mathrm{r}}, \theta_{\mathrm{r}}\right)=\frac{\mathrm{d} L_{\mathrm{r}}\left(\varphi_{\mathrm{r}}, \theta_{\mathrm{r}}\right)}{\mathrm{d} E_{\mathrm{i}}\left(\varphi_{\mathrm{i}}, \theta_{\mathrm{i}}\right)}
$$

ist eine charakteristische Eigenschaft eines Punktes auf der Oberfläche einer Probe. Dabei sind $E_{\mathrm{i}}$ in $\mathrm{Wm}^{-2}$ die Bestrahlungsstärke auf dem Oberflächenpunkt und $L_{\mathrm{r}}$ in $\mathrm{Wm}^{-2} \mathrm{sr}^{-1}$ die Strahldichte des reflektierten Lichts. Die Azimute $\varphi$ und die Polarwinkel $\theta$ beschreiben die Richtungen des einfallenden (Index $i$ ) und des reflektierten (Index $r$ ) Lichts (Abb. 1).

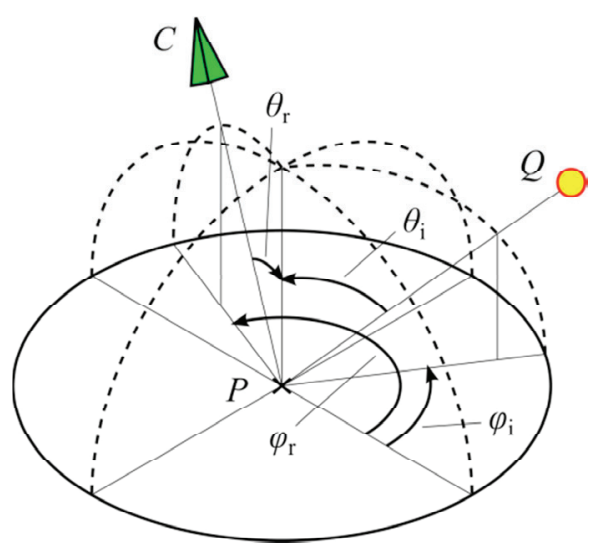

Abb. 1: Parametrisierung von einfallendem und reflektiertem Licht bei einer Gonioreflektometer-Anordnung
Ein Gonioreflektometer ist ein Gerät zum Messen der BRDF und besteht im Allgemeinen aus einer Lichtquelle (1) am Punkt $Q$, einem lichtempfindlichen Detektor (2) mit dem optischen Zentrum $C$ und einer Aufnahme für die Probe (3) mit dem auf ihr liegenden, beobachteten Punkt $P$ [2]. Eine mechanische Konstruktion ermöglicht die drei Komponenten relativ zueinander so zu bewegen, dass die interessierenden Winkelkombinationen erreicht werden können. Um die vier Winkel $\varphi_{\mathrm{i}}, \theta_{\mathrm{i}}, \varphi_{\mathrm{r}}$ und $\theta_{\mathrm{r}}$ unabhängig einstellen zu können, benötigt man mindestens vier mechanische Freiheitsgrade (DOFs) [3].

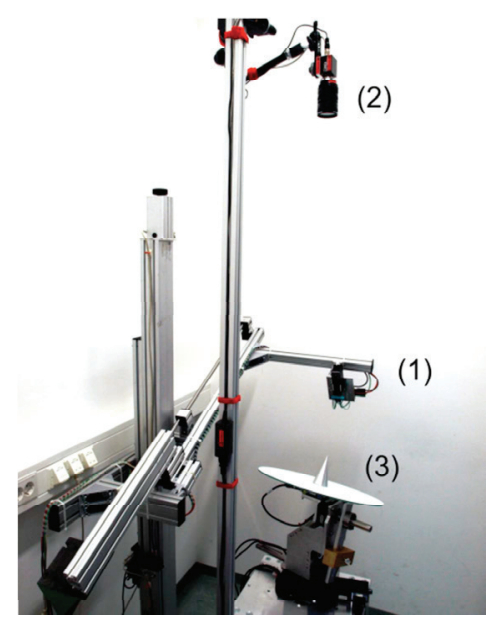

Abb. 2: Aufbau des Gonioreflektometers. Auf dem sphärischen Gelenk ist hier das Kegel-Kalibriertarget montiert. 
Ziel ist, mithilfe der gemessenen BRDF von metallischen Oberflächen auf Oberflächeneigenschaften wie Abweichungen von der mittleren Rauigkeit zurückzuschließen und Defekte wie mikroskopisch kleine Kratzer und Dellen zu detektieren.

Dazu wurde im Labor ein Gonioreflektometer aufgebaut (Abb. 2). Es besteht aus einer Hochleistungs-LED (XQ-E Serie von Cree) als Lichtquelle (1), die entlang eines Halbkreisbogens bewegt werden kann, einer fix im Raum montierten Kamera (GC1600H von Allied Vision Technologies $\mathrm{GmbH}$ ) mit einem Zoomobjektiv (Pentax 8-48mm) als Detektor (2) und einem mit drei Rotationstischen von Newport aufgebauten sphärischen Gelenk (3) zum Bewegen der Probe.

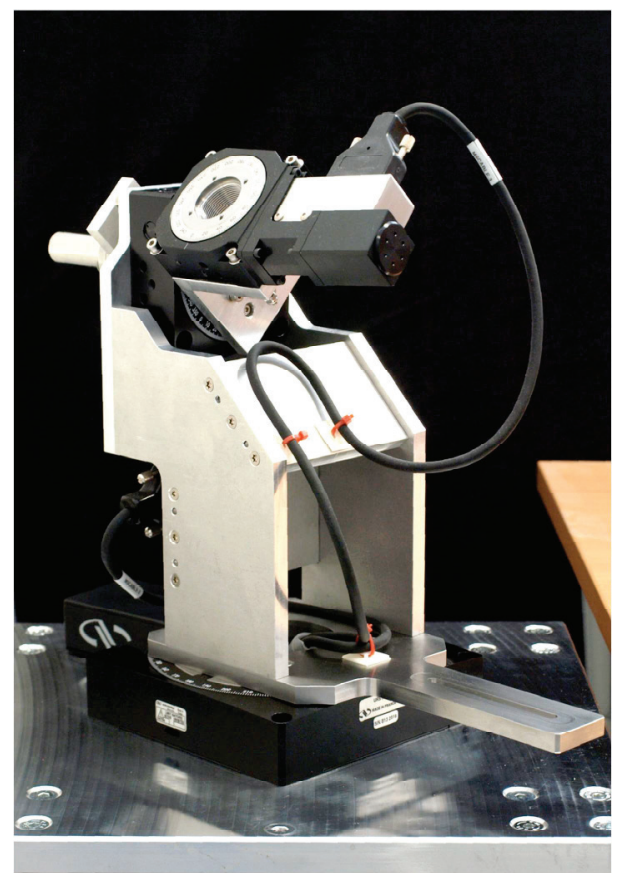

Abb. 3: Das sphärische Gelenk erlaubt, eine Probe entlang einer Kugelfläche $z u$ bewegen.

Zusammen realisieren die bewegliche Lichtquelle und das sphärische Gelenk vier DOFs. Mit der Kamera ist es möglich, mit einer einzelnen Aufnahme mehrere Punkte auf der Oberfläche zu analysieren, und die relativ kleine LED $\left(1.6 \times 1.6 \mathrm{~mm}^{2}\right)$ ist eine gute Näherung einer Punktlichtquelle.

Für die Funktion des Gonioreflektometers müssen die geometrischen Beziehungen zwischen den einzelnen Komponenten, sowie das Abbildungsverhalten des optischen Systems bekannt sein. Da sich diese Verhältnisse beim Laboraufbau potentiell ändern können (z.B. durch Objektivwechsel) und dadurch wiederholte Kalibrierungen notwendig sind, wird ein möglichst automatisierter Kalibriervorgang angestrebt. Dieser wird mit zwei eigens ange- fertigten, jeweils auf dem sphärischen Gelenk montierten Kalibriertargets durchgeführt und ist dementsprechend in zwei Teile gegliedert:

1. Mit einem Schachbrett-Kalibriertarget werden die Konstruktionsparameter des sphärischen Gelenks (Abschnitt 2.2), die intrinsischen Parameter des optischen Systems (Abschnitt 2.3), sowie relative Lage und Orientierung zwischen der Kamera und dem Gelenk (Abschnitt 2.4) ermittelt.

2. In einem weiteren Kalibriervorgang werden einzelne Positionen der Lichtquelle und somit die Kreisbogentrajektorie, auf der sich die Lichtquelle bewegen kann, bestimmt (Abschnitt 3).

\section{Kalibrierung von sphärischem Gelenk und Kamera}

Das Schachbrett-Kalibriertarget (Abb. 7) besteht aus einer $300 \times 300 \mathrm{~mm}^{2}$ großen Glasplatte, auf die ein mit einem Schachbrettmuster (8 × 5 Quadrate) bedrucktes Blatt Papier im A4-Format aufgeklebt wurde.

Bei einem Kalibriervorgang wird das Target automatisiert in verschiedene Posen gebracht und fotografiert. Mithilfe eines mathematischen Modells (Abschnitte 2.1 - 2.4) können die Koordinaten der Gitterpunkte des Schachbrettmusters in den aufgenommenen Bildern berechnet werden.

Die Parameter des Vorwärtsmodells werden schließlich nach der Methode der kleinsten Fehlerquadrate so geschätzt, dass die berechneten Gitterpunkte möglichst auf den in den Aufnahmen detektierten Punkten liegen.

\subsection{Koordinatentransformation}

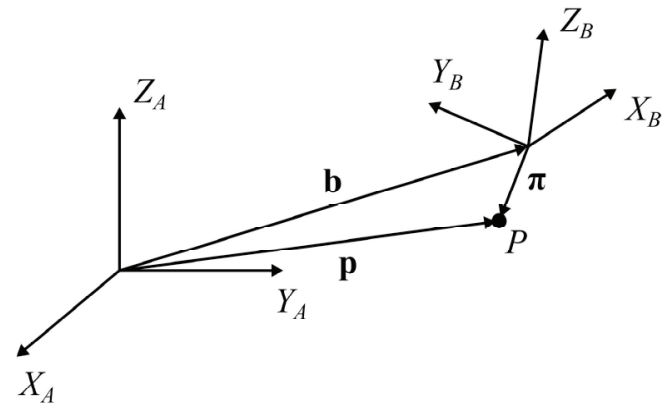

Abb. 4: Transformation des Ortsvektors von $P$ zwischen zwei rechtwinkeligen Koordinatensystemen

Um die Ortsvektoren eines Punktes $P$ zwischen zwei rechtwinkeligen Koordinatensystemen $F_{A}$ und $F_{B}$ umzurechnen (Abb. 4), kann die Koordinatentransformation [4]

$$
[\mathbf{p}]_{A}=[\mathbf{b}]_{A}+[\boldsymbol{\pi}]_{A}=[\mathbf{b}]_{A}+\mathbf{Q}[\boldsymbol{\pi}]_{B}
$$

verwendet werden. Dabei ist $\mathbf{Q}$ die Rotationsmatrix, die die Basisvektoren von $F_{A}$ parallel zu 
denen von $F_{B}$ ausrichtet und $\mathbf{b}$ ist der Vektor vom Ursprung von $F_{A}$ zum Ursprung von $F_{B}$. $\mathbf{Q}$ und $\mathbf{b}$ sind jeweils durch drei Parameter bestimmt, die Transformation $\{\mathbf{Q}, \mathbf{b}\}$ hat somit im allgemeinsten Fall sechs Freiheitsgrade.

\subsection{Das sphärische Gelenk}

Das sphärische Gelenk besteht aus drei Rotationstischen, die so der Reihe nach miteinander verbunden sind, dass sich ihre Rotationsachsen in einem Punkt schneiden. Die drei Tische bilden einen seriellen Roboter mit drei Gelenken und $i=1 \ldots 4$ Gliedern. Um die Geometrie des Roboters zu beschreiben, wird je ein Koordinatensystem $\mathcal{F}_{i}=\left\{X_{i}, Y_{i}, Z_{i}\right\}$ mit einem Glied fest verbunden. $\mathcal{F}_{1}$ ist mit dem nicht rotierenden Teil von Tisch eins verbunden, ist raumfest und wird als Basiskoordinatensystem bezeichnet. $\mathcal{F}_{4}$ ist mit dem rotierenden Teil von Tisch drei verbunden und wird als Endeffektorkoordinatensystem bezeichnet.

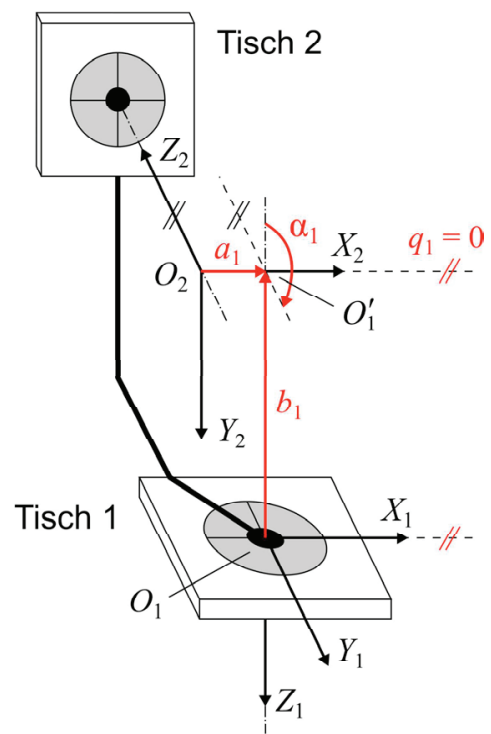

Abb. 5: Schematische Darstellung der Zusammenhänge zwischen den Koordinatensystemen $\mathcal{F}_{1}$ und $\mathcal{F}_{2}$ in Ausgangslage $\left(q_{1}=q_{1,0}+\Delta q_{1}=0\right) . \mathcal{F}_{3}$ und $\mathcal{F}_{4}$ folgen in analoger Weise.

Die Beziehungen zwischen den Koordinatensystemen lassen sich mit einer Variante der Denavit-Hartenberg (DH) Notation [5] beschreiben (Abb. 5). Die dabei vorgenommenen Modifikationen gegenüber der ursprünglichen DH Notation (siehe Regeln 2 und 3 in der folgenden Aufzählung) sind notwendig, um numerische Singularitäten bei der iterativen $\mathrm{Pa}$ rameterschätzung mit der Methode der kleinsten Fehlerquadrate zu vermeiden:

1. $Z_{i}$ ist die Achse des $i$-ten Gelenks. Die positive Richtung kann prinzipiell beliebig festgelegt werden und wird so gewählt, dass eine positive Drehwinkelvorgabe für den Tisch zu einer positiven Drehung im Sinne der Korkenzieherregel führt.

2. $X_{i}$ ist die gemeinsame Normale von $Z_{i-1}$ und $Z_{i}$. Die positive Richtung wird durch die Drei-Finger-Regel $\left(Z_{i-1} \rightarrow Z_{i} \rightarrow X_{i}\right)$ festgelegt (Bei der ursprünglichen DH Notation weist $X_{i}$ immer von $O_{i-1}^{\prime}$ nach $O_{i}$ ).

3. Die $X_{i+1}$-Koordinate von $O_{i}^{\prime}$ ist $a_{i}$ (Bei der ursprünglichen $\mathrm{DH}$ Notation ist $a_{i}$ der Normalabstand zwischen $Z_{i}$ und $Z_{i+1}$ und daher stets positiv).

4. Die $Z_{i}$-Koordinate von $O_{i}^{\prime}$ ist $b_{i}$.

5. Der Winkel zwischen $Z_{i}$ und $Z_{i+1}$, gemessen um die positive Richtung von $X_{i+1}$, ist $\alpha_{i}$.

6. Der Winkel zwischen $X_{i}$ und $X_{i+1}$, gemessen um die positive Richtung von $Z_{i}$, ist $q_{i}$.

Je Gelenk sind so die konstanten Gelenkparameter $a_{i}, b_{i}$ und $\alpha_{i}$ und die Gelenkvariable $q_{i}$ definiert. Letztere setzt sich dabei aus dem konstanten Anteil $q_{i, 0}$, der sich aus dem Montagewinkel ergibt, und dem veränderlichen Anteil $\Delta q_{i}$, der dem eingestellten Gelenkwinkel entspricht, zusammen:

$$
q_{i}=q_{i, 0}+\Delta q_{i} .
$$

Es kann gezeigt werden [4], dass sich entsprechend der modifizierten DH Notation die einzelnen Transformationen (Gl. (2)) zu

$$
\mathbf{Q}_{i}=\left(\begin{array}{ccc}
\cos q_{i} & -\lambda_{i} \sin q_{i} & \mu_{i} \sin q_{i} \\
\sin q_{i} & \lambda_{i} \cos q_{i} & -\mu_{i} \cos q_{i} \\
0 & \mu_{i} & \lambda_{i}
\end{array}\right)
$$

und

$$
\mathbf{b}_{i}=\left(\begin{array}{c}
-a_{i} \cos q_{i} \\
-a_{i} \sin q_{i} \\
b_{i}
\end{array}\right)
$$

mit $\lambda_{i}=\cos \alpha_{i}$ und $\mu_{i}=\sin \alpha_{i}$ ergeben.

Die gesamte Transformation $\left\{\mathbf{Q}_{41}, \mathbf{b}_{41}\right\}$ von $F_{4}$ nach $F_{1}$ ist dann mit

$$
\mathbf{Q}_{41}=\mathbf{Q}_{1} \mathbf{Q}_{2} \mathbf{Q}_{3} \mathbf{Q}_{4}
$$

und

$$
\mathbf{b}_{41}=\mathbf{b}_{1}+\mathbf{Q}_{1} \mathbf{b}_{2}+\cdots+\mathbf{Q}_{1} \mathbf{Q}_{2} \mathbf{Q}_{3} \mathbf{b}_{4}
$$

gegeben.

Zu den oben genannten Regeln gelten die folgenden beiden Ausnahmen für $\mathcal{F}_{1}$ und $\mathcal{F}_{4}$ :

1. $O_{1}$ muss auf $Z_{1}$ liegen, ansonsten kann $\mathcal{F}_{1}$ beliebig gewählt werden. Dementsprechend werden $b_{1}=-100 \mathrm{~mm}$ (die $X_{1} Y_{1}$ Ebene liegt somit ungefähr auf der Basisplatte, auf der das sphärische Gelenk montiert ist) und $q_{1,0}=0^{\circ}$ festgelegt.

2. $\mathcal{F}_{4}$ kann, der Anwendung entsprechend, beliebig gewählt werden. Es werden daher 
der Einfachheit halber $a_{3}=b_{3}=0 \mathrm{~mm}$ und $\alpha_{3}=q_{3,0}=0^{\circ}$ gewählt.

Somit verbleiben für das sphärische Gelenk die sechs zu schätzenden Konstruktionsparameter $a_{1}, \alpha_{1}, a_{2}, \alpha_{2}, b_{2}$ und $q_{2,0}$.

\subsection{Das Kameramodell}

Mit der Kamera ist das räumliche Koordinatensystem $\mathcal{F}_{\mathrm{C}}=\left\{X_{\mathrm{C}}, Y_{\mathrm{C}}, Z_{\mathrm{C}}\right\}$ fest verbunden. Der Ursprung von $\mathcal{F}_{C}$ befindet sich im optischen Zentrum $C$, und $Z_{C}$ ist die optische Achse und weist vom Bildsensor weg (Abb. 6).

Ausgehend vom idealisierten Lochkameramodell wird ein Punkt mit Ortsvektor $\mathbf{x}=(x, y, z)^{T}$ mittels Zentralprojektion [6] auf

$$
\widetilde{\mathbf{p}}=\left(\begin{array}{l}
\tilde{u} \\
\tilde{v}
\end{array}\right)=\frac{1}{z}\left(\begin{array}{l}
x \\
y
\end{array}\right)
$$

in der normierten Bildebene abgebildet [7]. Die normierte Bildebene mit dem Koordinatensystem $\{\widetilde{U}, \widetilde{V}\}$ befindet sich im Abstand 1 (einheitenlos) von $C$ entfernt, hat keine physikalische Entsprechung und dient als rechnerische Hilfsebene.

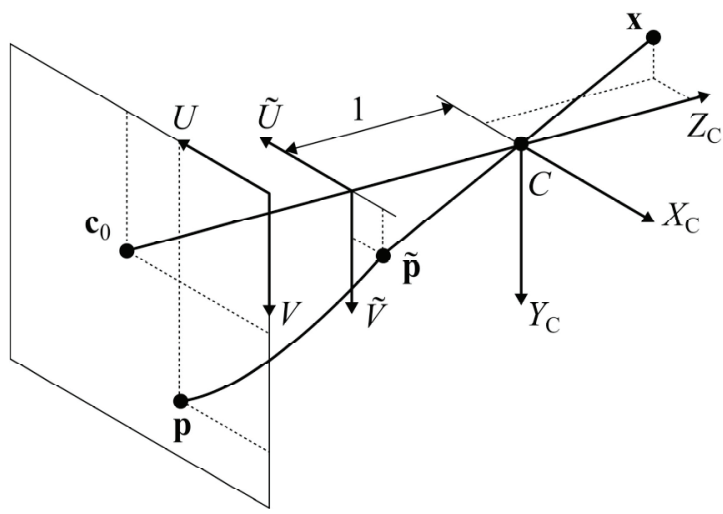

Abb. 6: Der Objektpunkt mit Ortsvektor $\mathbf{x}$ wird in einem rechnerischen Zwischenschritt mittels Zentralprojektion auf $\widetilde{\mathbf{p}}$ in der normierten Bildebene und danach mithilfe nicht linearer Terme auf p am Bildsensor abgebildet.

In weiterer Folge wird mithilfe nicht linearer Terme die Abbildung auf den Punkt $\mathbf{p}$ am Kamerasensor mit dem Bildkoordinatensystem $\mathcal{F}_{I}=\{U, V\}$ beschrieben. Dabei berücksichtigt der Korrekturterm

$$
\Delta^{(r)}=\left(k_{1} r_{\tilde{p}}^{2}+k_{2} r_{\tilde{p}}^{4}\right) \widetilde{\mathbf{p}}
$$

mit $r_{\tilde{p}}{ }^{2}=\sqrt{\tilde{u}^{2}+\tilde{v}^{2}}$ und den einheitenlosen Koeffizienten $k_{1}$ und $k_{2}$ den radialen Abbildungsfehler durch das Objektiv, der sich durch kissen- oder tonnenförmige Verzeichnung bemerkbar macht. Der Term

$$
\Delta^{(t)}=\left(\begin{array}{l}
2 t_{1} \tilde{u} \tilde{v}+t_{2}\left(r_{\tilde{p}}^{2}+2 \tilde{u}^{2}\right) \\
t_{1}\left(r_{\tilde{p}}^{2}+2 \tilde{v}^{2}\right)+2 t_{2} \tilde{u} \tilde{v}
\end{array}\right)
$$

mit den einheitenlosen Koeffizienten $t_{1}$ und $t_{2}$ beschreibt tangentiale Abbildungsfehler, die hauptsächlich durch eine nicht-perfekte Ausrichtung der optischen Komponenten im Objektiv hervorgerufen werden.

Mit der Kameramatrix

$$
\mathbf{K}=\left(\begin{array}{ccc}
f_{u} & \alpha_{c} f_{u} & u_{0} \\
0 & f_{v} & v_{0} \\
0 & 0 & 1
\end{array}\right)
$$

und unter Zuhilfenahme homogener Koordinaten [6] lassen sich schließlich die Komponenten des Ortsvektors $\mathbf{p}=(u, v)^{T}$ in px im aufgenommenen Bild berechnen:

$$
\left(\begin{array}{l}
u \\
v \\
1
\end{array}\right)=\mathbf{K}\left(\begin{array}{c}
\widetilde{\mathbf{p}}+\Delta^{(r)}+\Delta^{(t)} \\
1
\end{array}\right) .
$$

Mit den beiden Brennweiten $f_{u}$ und $f_{v}$ in px und dem einheitenlosen Schrägungsfaktor $\alpha_{c}$ werden parallelogrammförmige Sensorzellen berücksichtigt und $\mathbf{c}_{0}=\left(u_{0}, v_{0}\right)^{T}$ ist der Ortsvektor des Punktes in $\mathcal{F}_{I}$, an dem die optische Achse den Kamerachip schneidet. Für das optische System verbleiben somit neun zu schätzende intrinsische Parameter [8].

\subsection{Die gesamte Vorwärtsfunktion}

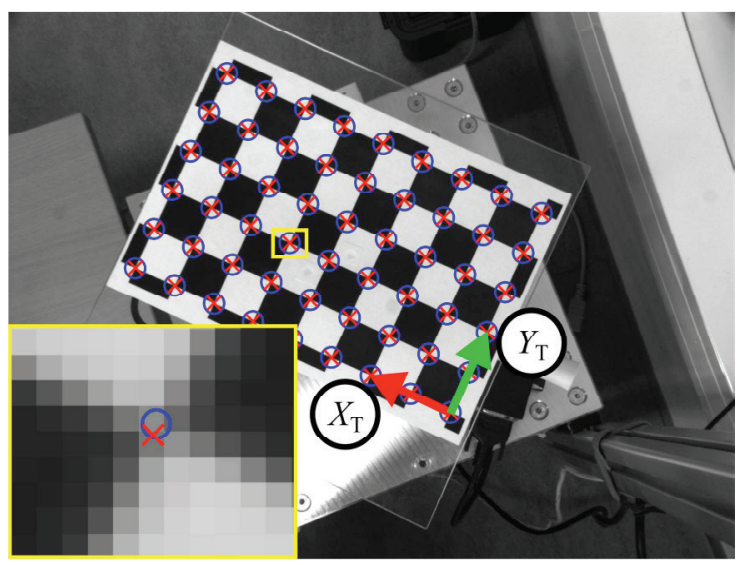

Abb. 7: Die detektierten (Kreise) und rückprojizierten (Kreuze) Gitterpunkte in einer Aufnahme des SchachbrettKalibriertargets

Es ist notwendig, dass das Schachbrett-Target in eine Richtung eine ungerade Anzahl von Gitterpunkten und in die andere Richtung eine gerade Anzahl aufweist, denn nur so kann damit das Target-Koordinatensystem $\mathcal{F}_{\mathrm{T}}=$ $\left\{X_{\mathrm{T}}, Y_{\mathrm{T}}, Z_{\mathrm{T}}\right\}$ eindeutig definiert werden: Der Ursprung befindet sich dann in der Ecke mit einem vollen schwarzen Quadrat, in der die ungerade Anzahl an Gitterpunkten im Uhrzei- 
gersinn folgt. In diese Richtung liegt die $X_{\mathrm{T}^{-}}$ Achse und entlang der Seite gegen den Uhrzeigersinn liegt die $Y_{\mathrm{T}}$-Achse (Abb. 7), die $Z_{\mathrm{T}^{-}}$ Achse weist in das Target hinein.

Die Gitterpunkte sind in $\mathcal{F}_{\mathrm{T}}$-Koordinaten bekannt und die gesamte Vorwärtsfunktion, die diese Punkte in Bildkoordinaten transformiert, setzt sich aus folgenden Teilschritten zusammen:

1. Transformation $\left\{\mathbf{Q}_{\mathrm{T} 4}, \mathbf{b}_{\mathrm{T} 4}\right\}$ entsprechend Gl. (2) von $\mathcal{F}_{\mathrm{T}}$ ins Endeffektorkoordinatensystem $\mathcal{F}_{4}$ (6 Parameter)

2. Transformation $\left\{\mathbf{Q}_{41}, \mathbf{b}_{41}\right\}$ entsprechend den Gleichungen (6) und (7) ins Basiskoordinatensystem $\mathcal{F}_{1}$ (6 Parameter)

3. Transformation $\left\{\mathbf{Q}_{1 \mathrm{C}}, \mathbf{b}_{1 \mathrm{C}}\right\}$ ins Kamerakoordinatensystem $\mathcal{F}_{\mathrm{C}}$ (6 Parameter)

4. Transformation ins Bildkoordinatensystem $\mathcal{F}_{I}$ entsprechend Gl. (12) (9 Parameter)

Insgesamt ergeben sich somit $27 \mathrm{zu}$ schätzende Parameter.

\subsection{Ergebnisse der Kalibrierung}

Bei einem exemplarischen Kalibriervorgang wurden 216 Bilder des SchachbrettKalibriertargets für verschiedene Kombinationen der Gelenkwinkel $\Delta q_{1}, \Delta q_{2}$ und $\Delta q_{3}$ aufgenommen. In 197 der 216 Bilder konnten alle Gitterpunkte mit der MATLAB-Funktion detectCheckerboardPoints erfolgreich detektiert werden (Abb. 7). Diese 197 Aufnahmen wurden dann für den iterativen Schätzalgorithmus nach der Methode der kleinsten Fehlerquadrate herangezogen (MATLABFunktion 1 sqnon 1 in).

In Tab. 1 sind von den 27 geschätzten Parametern die sechs Konstruktionsparameter des sphärischen Gelenks aufgelistet, da diese einen direkten Vergleich mit den zu erwartenden Werten eines idealen Gelenks erlauben.

Tab. 1: Gegenüberstellung der geschätzten Werte mit den Werten für ein ideales sphärisches Gelenk

\begin{tabular}{c|c|c} 
Parameter & geschätzt & ideal \\
\hline$a_{1}$ & $0.72 \mathrm{~mm}$ & $0 \mathrm{~mm}$ \\
\hline$\alpha_{1}$ & $89.99^{\circ}$ & $90^{\circ}$ \\
\hline$a_{2}$ & $0.03 \mathrm{~mm}$ & $0 \mathrm{~mm}$ \\
\hline$b_{2}$ & $-0.25 \mathrm{~mm}$ & $0 \mathrm{~mm}$ \\
\hline$\alpha_{2}$ & $90.06^{\circ}$ & $90^{\circ}$ \\
\hline$q_{2,0}$ & $179.1^{\circ}$ & $180^{\circ}$
\end{tabular}

Die drei Rotationsachsen des Gelenks schneiden sich genau dann in einem Punkt, wenn $a_{1}$, $a_{2}$ und $b_{2}$ je $0 \mathrm{~mm}$ sind. Außerdem stehen aufeinanderfolgende Rotationsachsen idealerweise jeweils normal aufeinander $\left(\alpha_{1}=\alpha_{2}=\right.$ $\left.90^{\circ}\right)$. Der zu erwartende Parameter $q_{2,0}=180^{\circ}$ ergibt sich aus der Montage der Rotationstische zwei und drei.

Die geschätzten Parameter entsprechen sehr gut den erwarteten Werten. Dies spricht einerseits für einen gut funktionierenden Kalibriervorgang und andererseits für ein sphärisches Gelenk, das in guter Näherung dem Ideal entspricht.

Ein weiteres Indiz für eine gute Schätzung ist der geringe mittlere Rückprojektionsfehler: Die mit Hilfe der insgesamt 27 geschätzten Parameter in die 197 Aufnahmen rückprojizierten Gitterpunkte (Abb. 7) sind im Mittel $0.46 \mathrm{px}$ von den detektierten Gitterpunkten entfernt.

\section{Schätzen der Lichtposition}

Die Lichtquelle des Gonioreflektometers kann entlang einer Kreisbogentrajektorie bewegt werden. Um die Lage im Raum und den Radius dieses Kreisbogens schätzen zu können, müssen drei oder mehr Punkte der Trajektorie bekannt sein. Dazu wird die Lichtquelle an mehrere Punkte der Trajektorie bewegt und es wird für jeden dieser Punkte die Position der Lichtquelle mithilfe von Aufnahmen des auf dem sphärischen Gelenk montierten KegelKalibriertargets geschätzt.

Das Kegel-Kalibriertarget besteht aus einer runden, mit weißer Folie beschichteten Glasscheibe mit $400 \mathrm{~mm}$ Durchmesser und einem darauf mittig montierten Aluminiumkegel mit $50 \mathrm{~mm}$ Grundkreisdurchmesser und $98.3 \mathrm{~mm}$ Höhe (Abb. 10).

\subsection{Das Verfahren}

Die Lichtquelle beleuchtet das KegelKalibriertarget und der Aluminiumkegel wirft einen Schatten auf die weiß beschichtete Glasplatte (Abb. 8).

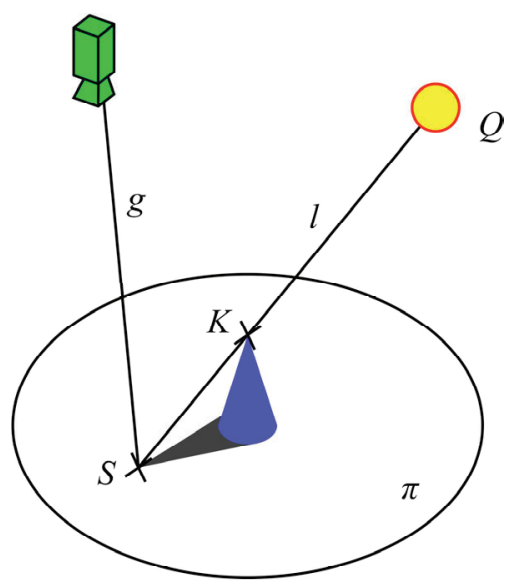

Abb. 8: Die geometrischen Verhältnisse beim Schätzen der Lichtposition mit dem Kegel-Kalibriertarget 
Die Gleichung der Ebene $\pi$, die die Oberfläche der Glasplatte beschreibt, sowie die Koordinaten der Spitze des Kegels $K$, beide in Abhängigkeit der Gelenkwinkel $\Delta q_{1}, \Delta q_{2}$ und $\Delta q_{3}$, sind aufgrund der vorangegangenen Kalibrierung des sphärischen Gelenks (Abschnitt 2) im Basiskoordinatensystem $F_{1}$ bekannt. Nach Detektion der Schattenspitze im aufgenommenen Bild (Abschnitt 3.2) kann mithilfe der Kamerakalibrierung die Darstellung der Geraden $g$ im Raum (Basiskoordinatensystem $\mathcal{F}_{1}$ ) aus den Bildkoordinaten abgeleitet werden. Die Schattenspitze in $\mathcal{F}_{1}$ erhält man nun als den Schnittpunkt $S=g \cap \pi$ und die beiden Punkte $S$ und $K$ definieren schließlich eine Gerade $l$, die durch die Lichtquelle $Q$ verläuft.

Bringt man das Kegel-Kalibriertarget mit dem sphärischen Gelenk in verschiedene Posen und wiederholt das oben beschriebene Verfahren, erhält man mehrere Geraden $l_{i}$ mit $i=1 \ldots n$, die alle näherungsweise durch die Position $Q$ der Lichtquelle verlaufen. Diese Geraden können jeweils durch eine skalare Variable $z_{i}$ parametrisiert als

$$
l_{i}: \mathbf{x}_{i}=\mathbf{o}_{i}+z_{i} \mathbf{r}_{i}
$$

dargestellt werden, mit dem Ortsvektor $\mathbf{o}_{i}$ eines Punktes auf $l_{i}$ und dem Richtungsvektor $\mathbf{r}_{i}$ der Geraden.

Die Geraden $l_{i}$ schneiden sich im Allgemeinen nicht genau in einem Punkt. Daher wird nach dem Punkt mit Ortsvektor $\mathbf{x}_{\text {opt }}$ gesucht, der die Summe der quadrierten Normalabstände zu den einzelnen Geraden minimiert:

$$
\mathbf{x}_{\mathrm{opt}}=\arg \min _{\mathbf{x}} \sum_{i=1}^{n}\left\|\mathbf{n}_{i}-\mathbf{x}\right\|^{2} .
$$

Dabei sind $\mathbf{n}_{i}$ die Orthogonalprojektionen von $\mathbf{x}$ auf die Geraden $l_{i}$ (Abb. 9).

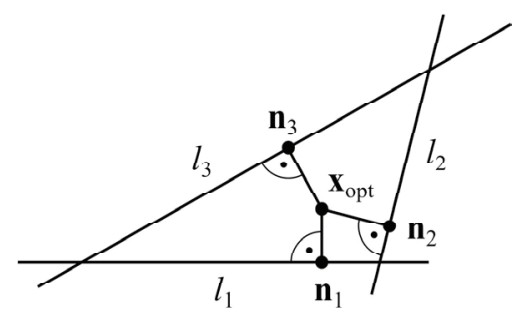

Abb. 9: $\quad x_{\text {opt }}$ minimiert die Summe der Quadrate der Normalabstände zu den Geraden $l_{i}$

Die Geradenparameter $z_{i}$, um die Orthogonalprojektionen $\mathbf{n}_{i}$ für $\mathbf{x}_{\mathrm{opt}} \mathrm{zu}$ berechnen, lassen sich zum Vektor $\mathbf{z}_{\text {opt }}$ zusammenfassen und es kann gezeigt werden [9], dass

$$
\mathbf{z}_{\text {opt }}=\mathbf{A}^{-1} \mathbf{d}
$$

gilt, wobei sich $\mathbf{A}$ als

$$
\mathbf{A}=\left(\begin{array}{cccc}
\left\|\mathbf{r}_{1}\right\|^{2} & 0 & \cdots & 0 \\
0 & \left\|\mathbf{r}_{2}\right\|^{2} & \cdots & 0 \\
\vdots & \vdots & \ddots & \vdots \\
0 & 0 & \cdots & \left\|\mathbf{r}_{n}\right\|^{2}
\end{array}\right)-\frac{1}{n} \mathbf{G}
$$

mit der Gramschen Matrix

$$
\mathbf{G}=\left(\begin{array}{cccc}
\mathbf{r}_{1}^{\mathrm{T}} \mathbf{r}_{1} & \mathbf{r}_{1}^{\mathrm{T}} \mathbf{r}_{2} & \cdots & \mathbf{r}_{1}^{\mathrm{T}} \mathbf{r}_{n} \\
\mathbf{r}_{2}^{\mathrm{T}} \mathbf{r}_{1} & \mathbf{r}_{2}^{\mathrm{T}} \mathbf{r}_{2} & \cdots & \mathbf{r}_{2}^{\mathrm{T}} \mathbf{r}_{n} \\
\vdots & \vdots & \ddots & \vdots \\
\mathbf{r}_{n}^{\mathrm{T}} \mathbf{r}_{1} & \mathbf{r}_{n}^{\mathrm{T}} \mathbf{r}_{2} & \cdots & \mathbf{r}_{n}^{\mathrm{T}} \mathbf{r}_{n}
\end{array}\right)
$$

darstellen lässt und

$$
\mathbf{d}=\left(\begin{array}{c}
\mathbf{r}_{1}^{\mathrm{T}}\left(\boldsymbol{\mu}-\mathbf{o}_{1}\right) \\
\mathbf{r}_{2}^{\mathrm{T}}\left(\boldsymbol{\mu}-\mathbf{o}_{2}\right) \\
\vdots \\
\mathbf{r}_{n}^{\mathrm{T}}\left(\boldsymbol{\mu}-\mathbf{o}_{n}\right)
\end{array}\right)
$$

mit

$$
\boldsymbol{\mu}=\frac{1}{n} \sum_{i=1}^{n} \mathbf{o}_{i}
$$

gilt. Der geschätzte Ortsvektor der Lichtquelle $Q$ ist dann

$$
\mathbf{x}_{\mathrm{opt}}=\frac{1}{n} \sum_{i=1}^{n} \mathbf{n}_{i}
$$

\subsection{Detektieren der Schattenspitze}

Eine korrekte Detektion der Schattenspitze in den Aufnahmen (Abb. 10) des KegelKalibriertargets ist ein essentieller Bestandteil für die Automatisierung des Verfahrens aus Abschnitt 3.1.

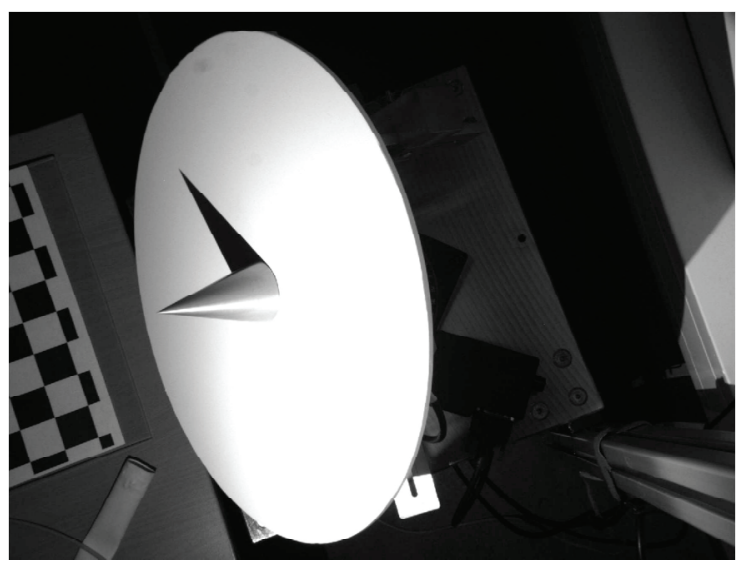

Abb. 10: Aufnahme des Kegel-Kalibriertargets

In einem ersten Schritt muss die Aufnahme mithilfe der intrinsischen Kameraparameter (Abschnitt 2.3) von Verzeichnungen befreit werden (Abb. 11). Das ist notwendig, damit die Kanten des Kegelschattens im Bild nicht gekrümmt erscheinen und so später gut mit einer Hough-Transformation detektiert werden können. 


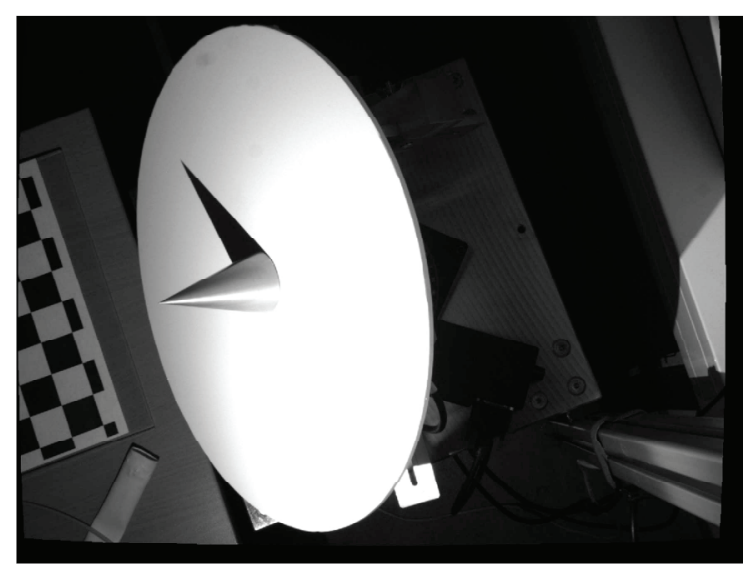

Abb. 11: Entzerrte Aufnahme

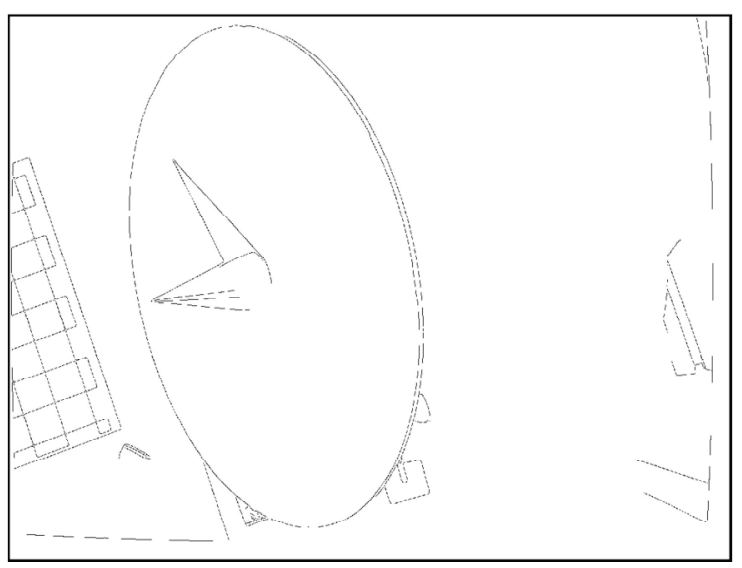

Abb. 12: Ergebnis der Kantendetektion

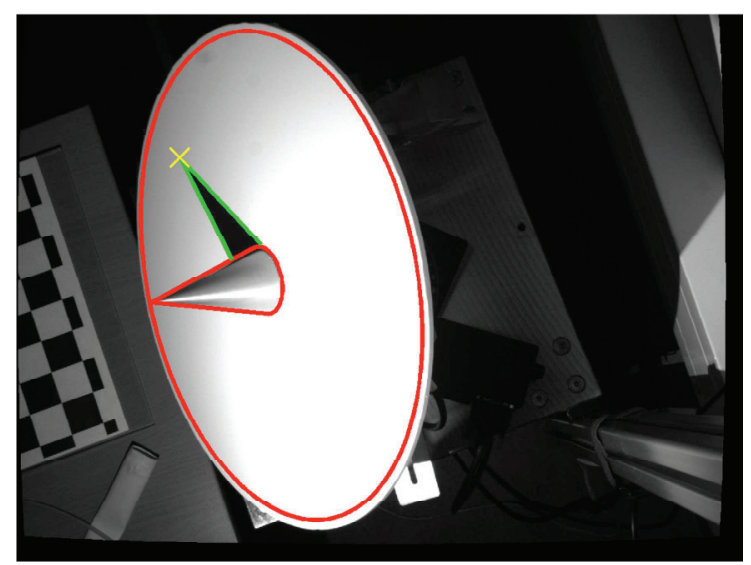

Abb. 13: Die Begrenzungen des runden Targets und des Kegel (rot), die mit der Hough-Transformation detektierten Schattenkanten (grün) und die Schattenspitze (gelbes Kreuz)

Danach werden alle Kanten im Bild mit dem Canny-Algorithmus [10] detektiert (MATLABBefehl edge, Abb. 12). Der Algorithmus verlangt zwei Schwellwerte: Mit dem unteren Schwellwert $T_{L}$ müssen die Kegelschattenkanten im Schwellwertbild vollständig enthalten sein und Bedingung für den oberen Schwellwert $T_{H}$ ist, dass zumindest ein Punkt jeder
Schattenkante, aber keine andere Kante in der weißen Fläche des Targets verbleibt. Gute Ergebnisse wurden mit $T_{L}=0.1$ und $T_{H}=0.2$ erzielt. Da die Schattenkanten aber einen hohen Kontrast zum sonst homogen weißen Target aufweisen, ist die Detektion auch gegenüber anders gewählten Schwellwerten potentiell robust. Der Canny-Algorithmus ist auch robust gegenüber unscharfen Schattenkanten, die durch eine nicht punktförmige Lichtquelle verursacht werden.

Als nächstes wird das Kantenbild mit der weiBen Fläche des Targets maskiert, damit nur die beiden Kanten des Kegelschattens verbleiben. Die Begrenzungen der verwendeten Maske, in Abhängigkeit der Gelenkwinkel $\Delta q_{1}, \Delta q_{2}$ und $\Delta q_{3}$, sind im Basiskoordinatensysem $\mathcal{F}_{1}$ bekannt und können mithilfe der in Abschnitt 2 geschätzten Parameter in die Aufnahmen projiziert werden (rote Linien in Abb. 13).

Den nun verbleibenden beiden Kanten des Kegelschattens können mit einer HoughTransformation [11] (MATLAB-Befehl hough) analytische Geradengleichungen zugeordnet werden und durch Schneiden dieser beiden Geraden erhält man schließlich die Bildkoordinaten der Schattenspitze.

\subsection{Ergebnisse der Positionsschätzung}

Bei einer exemplarischen Positionsschätzung wurden 96 Bilder des auf dem sphärischen Gelenk montierten Kegel-Kalibriertargets in verschiedenen Posen aufgenommen. Die Gelenkwinkel $\Delta q_{1}, \Delta q_{2}$ und $\Delta q_{3}$ wurden dabei so gewählt, dass die Schattenspitze näherungsweise drei konzentrische Kreise mit den Radien 50, 100 und $150 \mathrm{~mm}$ auf dem Target überstreicht, wenn man die 96 Posen durchläuft. Die resultierenden Geraden $l_{i}$ bilden somit drei gleichmäßig verteilte, einen Kegel beschreibenden Bündel. Die Gelenkwinkel dafür können mithilfe der geschätzten Parameter aus Abschnitt 2 berechnet werden, wenn die Position der Lichtquelle schon ungefähr bekannt ist (die dafür notwendige vorläufige Positionsschätzung wurde mit drei Aufnahmen des Targets in zufällig gewählten Posen durchgeführt). In 83 von den 96 Aufnahmen konnte die Schattenspitze erfolgreich detektiert werden. Bei den restlichen schlug die Detektion fehl, weil sich der Schatten im Bild zu einem großen Teil hinter dem Kegel befand. Die aus den 83 Aufnahmen geschätzte Position der Lichtquelle ist

$$
\mathbf{x}_{\text {opt }}=\left(\begin{array}{c}
281.9 \\
-3.3 \\
-404.4
\end{array}\right) \mathrm{mm}
$$

und die Standardabweichung der Orthogonalprojektionen $\mathbf{n}_{i}$ von $\mathbf{x}_{\mathrm{opt}}$ ist 


$$
\mathbf{s}_{\mathbf{n}_{i}}=\left(\begin{array}{l}
0.74 \\
1.04 \\
1.12
\end{array}\right) \mathrm{mm}
$$

Eine Interpretation von Gl. (22) ist, dass 95\% der Geraden $l_{i}$ einen nur 2.97 x $4.14 \times 4.48 \mathrm{~mm}^{3}$ großen Quader rundum $\mathbf{x}_{\text {opt }}$ durchlaufen. In Anbetracht der Relationen (die Lichtquelle hat etwa einen Abstand von 493mm zum sphärischen Gelenk) spricht dies für eine hohe Genauigkeit des vorgestellten Verfahrens.

\section{Resümee}

In Abschnitt 2 wurde ein Verfahren vorgestellt, um für den Laboraufbau eines Gonioreflektometers die intrinsischen Parameter des optischen Systems, die Konstruktionsparameter des sphärischen Gelenks und die relative Lage und Orientierung zwischen Kamera und sphärischem Gelenk zu schätzen. Das dazu auf dem Gelenk montierte SchachbrettKalibriertarget wird in verschiedene Positionen gebracht, fotografiert und anschließend werden die Aufnahmen zur Parameterschätzung ausgewertet. Alle Schritte funktionieren vollständig automatisiert und es sind keine Benutzerinteraktionen notwendig.

Das Schätzen der Position der Lichtquelle wurde in Abschnitt 3 beschrieben. Auch hierfür wird ein eigens angefertigtes KegelKalibriertarget in verschiedenen Posen fotografiert und es werden die Aufnahmen anschlieBend ausgewertet. Auch hier funktionieren alle Schritte vollständig automatisiert.

Die Automatisierung und der hohe Grad an Genauigkeit ermöglichen künftig, das Gonioreflektometer rasch und unkompliziert für neue geometrische Verhältnisse zu adaptieren (z.B. nach Änderung der Kameraposition) und legen so die Basis für die Messung der BRDF zur Überprüfung metallischer Oberflächen.

\section{Danksagung}

Diese Arbeit wurde im Rahmen des österreichischen COMET-K2 Zentrums des Linz Center of Mechatronics (LCM) durchgeführt und wurde von der österreichischen Bundesregierung und dem Land Oberösterreich finanziert.

\section{Literatur}

[1] F. E. Nicodemus, J. C. Richmond, J. J. Hsia, I. W. Ginsberg, and T. Limperis. Geometrical Considerations and Nomenclature for Reflectance. NBS Monograph. U.S. Government Printing Office, 1977.

[2] M. Haindl and J. Filip. Visual Texture. Accurate Material Appearance Measurement, Representation and Modeling. Advances in Computer Vision and Pattern Recognition. SpringerVerlag, London, 2013. ISBN 978-1-4471-49019.

[3] A. W. Winkler and B. G. Zagar. Building a gonioreflectometer - a geometrical evaluation. In 2015 IEEE International Instrumentation and Measurement Technology Conference (I2MTC 2015) Proceedings, pages 1900-1905, May 2015. ISBN 978-1-4799-6113-9.

[4] J. Angeles. Fundamentals of Robotic Mechanical Systems: Theory, Methods, and Algorithms. Mechanical Engineering Series. Springer International Publishing, 2013. ISBN 9783319018508.

[5] J. Denavit and R. S. Hartenberg. A kinematic notation for lower-pair mechanisms based on matrices. Trans. ASME E, Journal of Applied Mechanics, 22: 215-221, June 1955.

[6] R. I. Hartley and A. Zisserman. Multiple View Geometry in Computer Vision. Cambridge University Press, 2000.

[7] A. W. Winkler and B. G. Zagar. A curve fitting method for extrinsic camera calibration from a single image of a cylindrical object. Measurement Science and Technology, 24 (8): 084001, August 2013. ISSN 0957-0233. doi: 10.1088/0957-0233/24/8/084001.

[8] J. Heikkilä and O. Silvén. A four-step camera calibration procedure with implicit image correction. In CVPR '97 Proceedings of the 1997 Conference on Computer Vision and Pattern Recognition (CVPR '97), pages 1106- 1112. IEEE, IEEE Computer Society Washington, DC, USA @1997, June 1997. doi: 10.1109/CVPR.1997.609468.

[9] J.-Y. Bouguet. Visual Methods for ThreeDimensional Modeling. PhD thesis, California Institute of Technology, May 1999.

[10] J. Canny. A computational approach to edge detection. IEEE Trans. Pattern Anal. Mach. Intell., 8 (6): 679-698, June 1986. ISSN 01628828. doi: 10.1109/TPAMI.1986.4767851.

[11] R. C. Gonzalez and R. E. Woods. Digital Image Processing. Pearson Prentice Hall, 2008. 